\title{
Biochemical Parameters Alterations in Multiple Sclerosis: A Longitudinal Study and Review of the Literature
}

\author{
Nicola Salvatore Orefice ${ }^{1 *}$, Olga Ferraro ${ }^{2}$, Francesco Barbato ${ }^{2}$, Antonio Carotenuto ${ }^{2}$, \\ Roberta Lanzillo $^{2}$, Vincenzo Brescia Morra ${ }^{2}$, Giovanni Coppola ${ }^{2}$, Giuseppe Orefice ${ }^{2}$ \\ ${ }^{1}$ Department of Experimental Pharmacology, Federico II University of Naples, Italy; ${ }^{2}$ Department Neurological Sciences, Federico II \\ University of Naples, Italy. \\ Email: *nicolasalvatore.orefice@unina.it
}

Received January $10^{\text {th }}, 2012$; revised February $15^{\text {th }}, 2012$; accepted March $8^{\text {th }}, 2012$

\begin{abstract}
Multiple Sclerosis (MS) is a chronic inflammatory disease of the central nervous system (CNS) and is characterized by inflammation, demyelination and desctruction of oligodendrocytes and axons. The immunology of MS is complex, involving autoreactive Th1 and Th17 lymphocytes, cells of the innate immune system including dedritic, natural killer (NK) and microglia cells, as well as vascular endothelial cells. Multiple Sclerosis (MS) is based by a series of biochemical changes affecting to different extent neuronal functions; great attention has been focused on: liver enzymes, thyroid function tests, autoantibodies, lipid profile and uric acid levels. Following the introduction of therapy with drugs that modify the course of disease, more attention has been paid to the change of biochemical parameters, in particular to evaluate possible adverse effects. Aim of this study was to perform a review of the literature on biochemical alterations in treated and untreated MS patients and to assess laboratory parameters alterations in a cohort of MS patients, before and during treatment. On a total of 624 patients followed at our center, we selected those who practiced for the first time a therapy with IFN beta $1 \mathrm{a}$ at various doses, IFN beta $1 \mathrm{~b}$ and Glatiramer acetate, that were $595(95 \%)$, with baseline biochemical evaluation available in 488 (82\%) (307 females, mean age 36.6 years). We considered the evolution of various biochemical parameters during treatment. The following outcome variables were evaluated: laboratory changes, in particular liver function, thyroid function, lipids and blood cell count. Percentages were compared using the chisquare test. ANOVA was performed to evaluate changes of laboratory tests over time between therapy groups. Our finding confirm the presence of biochemical alterations at baseline, in particular regarding liver enzymes, thyroid hormones and lipids. Treatment effects were more evident on liver enzymes elevation, anti thyroid antibodies production and blood cholesterol decrease. These effects were mainly transient and self remitting without suspension of therapy. Biochemical alterations, mainly related to autoimmune pathology and lipid metabolism change were encountered. Treatments increased transient biochemical alterations particulary in patients with baseline changes, suggesting a stricter monitoring of blood exams in this category of patients.
\end{abstract}

Keywords: Multiple Sclerosis; Biochemical Alterations; Liver Enzymes; Thyroid; Lipids

\section{Introduction}

Multiple Sclerosis (MS) is a progressive, invalidating pathological state, the exact etiology of which is still uncertain [1]. It is considered as an autoimmune disease although the reasons for autoimmune disease although the reasons for the autoimmune demyelinization are far to be clear [2]. At the molecular level, MS is characterized by a series of biochemical changes affecting neuronal functions [3], some of which are in common with other neurodegenerations such as Alzheimer's [4] and Parkinson's disease [5]. Many clinical reports described changes in biochemical parameters in untreated multiple

*Corresponding author. sclerosis (MS) patients, in particular regarding liver enzymes, thyroid function tests, autoantibodies, lipid profile and uric acid levels. More recently the biochemical changes during the natural history of MS have become more difficult to assess, owing to the large availability of disease modifying drugs. Therefore we should refer to the pre-interferon (IFN) era studies or to baseline laboratory evaluations in cohorts of patients followed longitudinally on immunomodulatory therapies.

Hypertransaminasemia: autoimmune diseases are associated with an increased risk of hypertransaminasemia. In the course of MS, cases of autoimmune hepatitis and primary biliary cirrhosis have been reported [6]. Biochemical data collected in longitudinal studies on liver func- 
tion in the course of interferon therapy showed a subclinical hypertransaminasemia in $4.6 \%$ of patients already at basal [7].

Dysthyroidism: dysthyroidism and autoimmune thyroiditis are more frequent in the course of MS. In particular it was demonstrated that males with MS have the same prevalence of thyroid disorders as women, with or without MS [8]. At baseline alterations of thyroid hormones and/or thyroid stimulating hormon (TSH) were observed in $5.3 \%$ of patients [7].

Dyslipidemia: an increase in total cholesterol and HDL cholesterol levels were found in patients at onset of MS, without correlations between lipid levels and clinical parameters. In a study evaluating during 6 months followup with monthly brain MRI with gadolinium, Pozzilli et al. showed a correlation between total cholesterol and LDL cholesterol levels with the number of active plaques at brain MRI [9]. This study suggested the possibility of considering plasma cholesterol as a biological marker of disease activity in patients at onset of MS.

Changes of uric acid: in the course of MS a reduced value of uric acid (UA) was found, in normal range, compared to healthy subjects, in the absence of correlations with disease activity, duration, course or degree of disability [10]. This study favored the view that reduced UA in MS acts as a primary, constitutive loss of protection against oxidative agents. This reduction has been confirmed in other studies that showed also an inverse correlation with clinical and neuroradiological disease activity [11], while therapy with steroid boli for clinical relapses was associated with a significant increase in the values of UA compared to pre-treatment values [12].

\section{Changes in Biochemical Parameters during Therapy with Disease Modifying Drugs}

Following the introduction of therapy with drugs that modify the course of disease, more attention has been focused on biochemical parameters changes, particulary to evaluate possible adverse effects: Liver effects: a transient, but significant, increase in transaminases in $30 \%-40 \%$ of patients treated with IFNs was demonstrated, especially in the first six months of therapy [7]. Compared to pilot studies, these alterations were more obvious in post-marketing studies, but rarely so severe to lead to discontinuation of therapy. There seems to be a direct hepatotoxic effect of IFNs, with action on the P450 cytochrome [13].

Thyroid effects: alterations of thyroid hormones have been attributed to the Interferon in MS, but recently dysthyroidism did not appear significantly increased on IFN treatment compared to the risk of MS dependent comorbidity [7]. There seem to be disease related changes both in hypothyroidism and hyperthyroidism. The production of anti-thyroid auto antibodies without apparent clinical correlate is frequent. Transient dysthyroidism in not predisposed patients do not occur beyond the first year of therapy and it is therefore advisable to monitor thyroid parameters beyond the first year of therapy only in patients with a history of thyroiditis [14].

Hematological effects: dose-related cytopenias were observed in $10 \%$ of patients treated with IFNs, especially regarding the white cells. Severe leukopenia, lymphopenia and neutropenia are uncommon. These alterations are usually early (within 3 months), asymptomatic and not associated with increased risk of infection [15]. The phenomenon is entirely peripheral, with normal bone marrow, as a redistribution of blood cells.

Lipid profile changes: pro-inflammatory cytokines have a prominent role in determining changes in lipids metabolism. Several studies have emphasized that the effect of IFNs results in a decrease of cholesterol and increased triglycerides [16]. It is unclear whether IFN acts on lipid metabolism directly or through a different immunomodulatory mechanism.

Uric acid: during treatment with various immunomodulatory drugs currently on the market, there was evidence of increased levels of uric acid only with Glatiramer acetate, but not with Interferon. It has been suggested that the natural inhibitor of free radicals role of uric acid can intervene in the mechanism of action of Glatiramer acetate [17].

\section{Patients and Methods}

We considered the evolution of various biochemical parameters during treatment with IFN beta $1 \mathrm{a}$ at various doses, IFN beta $1 \mathrm{~b}$ and Glatiramer acetate. On a total of 624 patients followed at our center, those who practiced for the first time therapy with IFN or Glatiramer acetate were 595 (95\%), with baseline biochemical evaluation available in 488 (82\%) (307 females, mean age 36.6 years). Blood sampling was performed in the morning after a 12-hours fasting. The follow-up data of these patients were considered for statistical analysis. The following outcome variables were evaluated: laboratory changes, in particular liver function, thyroid function, lipids and blood cell count. Percentages were compared using the chi-square test.

\section{Statistical Analysis}

ANOVA was performed to evaluate changes of laboratory tests over time between therapy groups.

\section{Results}

\subsection{Baseline Alterations}

In the group of 488 de novo patients followed at our MS center, abnormal liver enzyme $(>40 \mathrm{U} / \mathrm{L})$ were present in 
3.5\% and $12.3 \%$ for AST and ALT respectively (Figure 1).

Thyroid function tests showed a $3.5 \%$ of patients with altered thyroid hormones, while anti thyroid antibodies with values $>100 \mathrm{U} / \mathrm{L}$ were present in $6.9 \%$ and $8.9 \%$ for anti-thyroglobulin ( $\mathrm{TgAb})$ and for anti-thyreoperoxidase (TPOAb) respectively (Figure 2).

In a subgroup of patients we focused attention on lipid profile: at baseline, total cholesterol ranged between 2.5 and $7.6 \mathrm{mmol} / \mathrm{L}$ (normal laboratory range: 2.4 - 5.5 $\mathrm{mmol} / \mathrm{L}$; desirable value: $<4.9 \mathrm{mmol} / \mathrm{L})$. LDL ranged between 0.7 and $5 \mathrm{mmol} / \mathrm{L}$ (normal laboratory range: 1.5 $3.9 \mathrm{mmol} / \mathrm{L}$; desirable value: $<3.4 \mathrm{mmol} / \mathrm{L}$ ).

\subsection{Follow-Up}

Patients were divided into five treatment groups (IFN beta 1a $30 \mathrm{mcg} 27.5 \%$, IFN beta 1 a $22 \mathrm{mcg} 17.6 \%$, IFN beta $1 \mathrm{a} 44 \mathrm{mcg} 13.5 \%$, IFN beta $1 \mathrm{~b} 26.6 \%$ and Glatiramer acetate $14.8 \%$ ). The mean follow-up was 25.1 months. We have documented a transient increase of liver enzymes in the first 6 - 12 months of therapy in all subgroups, statistically significant for the higher IFN dose regimens, with a tendency to return to baseline levels by the end of follow-up, without change of dosage of the drug (Figures 3 and 4). In particular, a peak of AST and ALT levels was registered at month 2 for IFN beta $1 \mathrm{~b}$ and at month 3 for the two s.c. IFN beta $1 \mathrm{a}$ formulations.

Anti-thyroglobulin antibodies with clinically significant values $(>100 \mathrm{U})$ increased and remained persistently high at 24 months of treatment with IFN beta 1 a 22

Overall percentage of patients with baseline

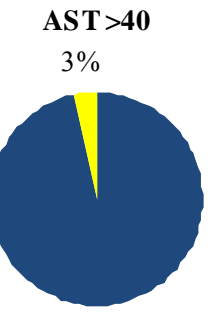

mcg $(13.3 \%)$ and with Glatiramer acetate $(23.5 \%)$ with values even higher than 5000 IU. High levels of antiTPO antibodies $(>100 \mathrm{U} / \mathrm{L})$ were detected at 24 months only in the group treated with Glatiramer acetate (14.3\%). Thyroid function analysis showed a transient increase in free-tri-iodothyronine (fT3) levels $(3.09 \mathrm{pg} / \mathrm{ml}, \mathrm{F}=2.25$, $\mathrm{P}<0.01)$ at the first month of therapy, without any clinical correlate, with spontaneous normalization.

The lipid profile, in particular, has been studied in a subgroup of 253 consecutive patients during treatment with Interferon Beta $1 \mathrm{a}$ or $1 \mathrm{~b}$, showing modest but significant decrease in total cholesterol (up to $-8 \%$ ) over 1 year follow-up, while for triglycerides was found only a slight and not significant increase (up to $+6 \%$ ). We have not revealed differences between the different IFN therapies in the influence on lipid values [18]. In the total sample, the total cholesterol level decreased significantly from month 3 and remained significantly lower than baseline levels throughout the follow-up with a pharmacological significant effect only for IFN beta $1 \mathrm{~b}(\mathrm{p}<$ 0.01 ), IFN beta 1 a $30 \mathrm{mcg}$ and IFN beta 1a $44 \mathrm{mcg}$ (p < 0.001 ), and a non significant trend with IFN beta 1a 22 mcg (Figure 5).

Triglycerides did not show any significant change. Patients with higher relapse rate during treatment did not show significantly higher cholesterol levels, contrarily to other literature evidences [9], not confirming, therefore, the role as a biochemical marker of disease activity suggested above for plasma cholesterol. The decrease of cholesterol levels was confirmed also on long term follow-up on IFN beta 1a therapy [19].

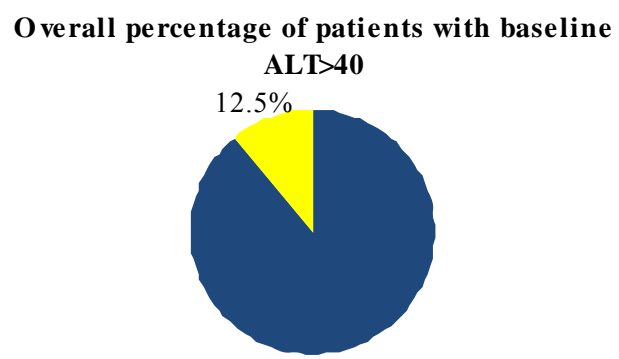

Figure 1. Overall percentage of patients with AST and ALT > 40 IU at baseline.

Overall percentage of patients with baseline TgAb $>100 \mathrm{U}$

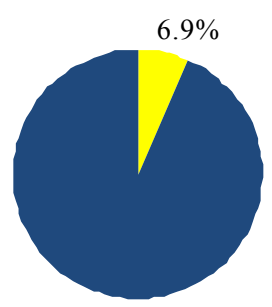

Overall percentage of patients with baseline TPO Ab > 100U

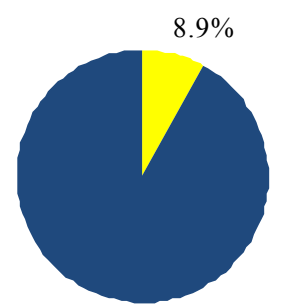

Figure 2. Overall percentage of patients with TgAb and TPOAb > $100 \mathrm{IU}$ at baseline. 


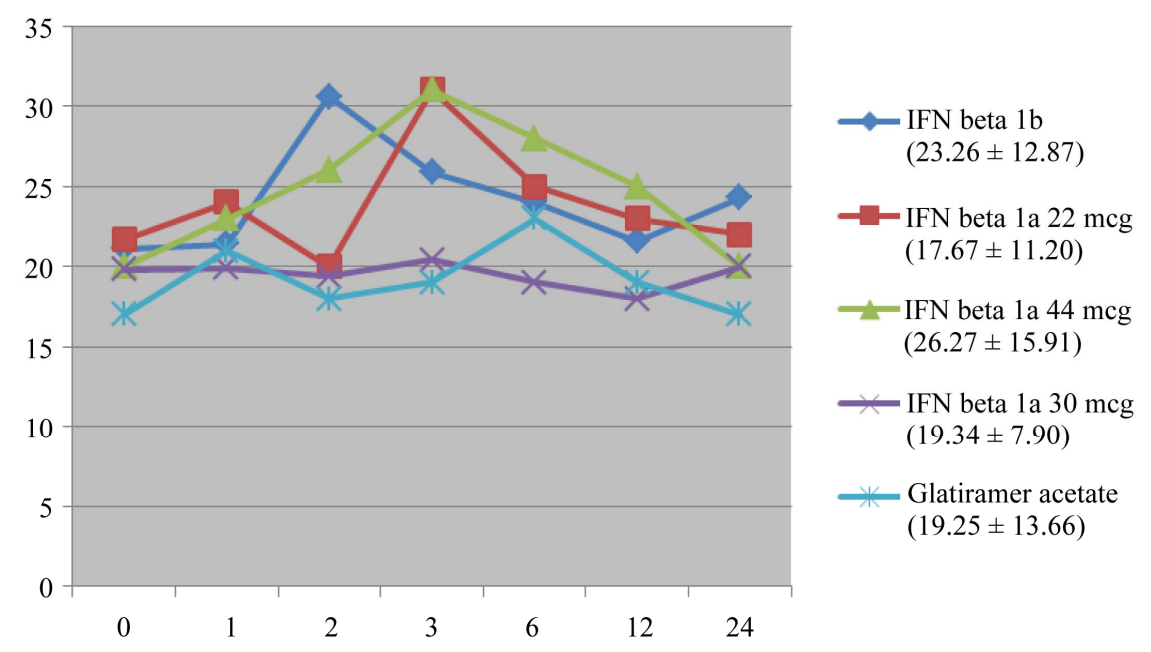

Figure 3. AST. Transient increase statistically significant for IFN beta 1 b, IFN beta 1a 22 mcg and 44 mcg (p $<0.01)$. Mean and SD of AST values in brackets.
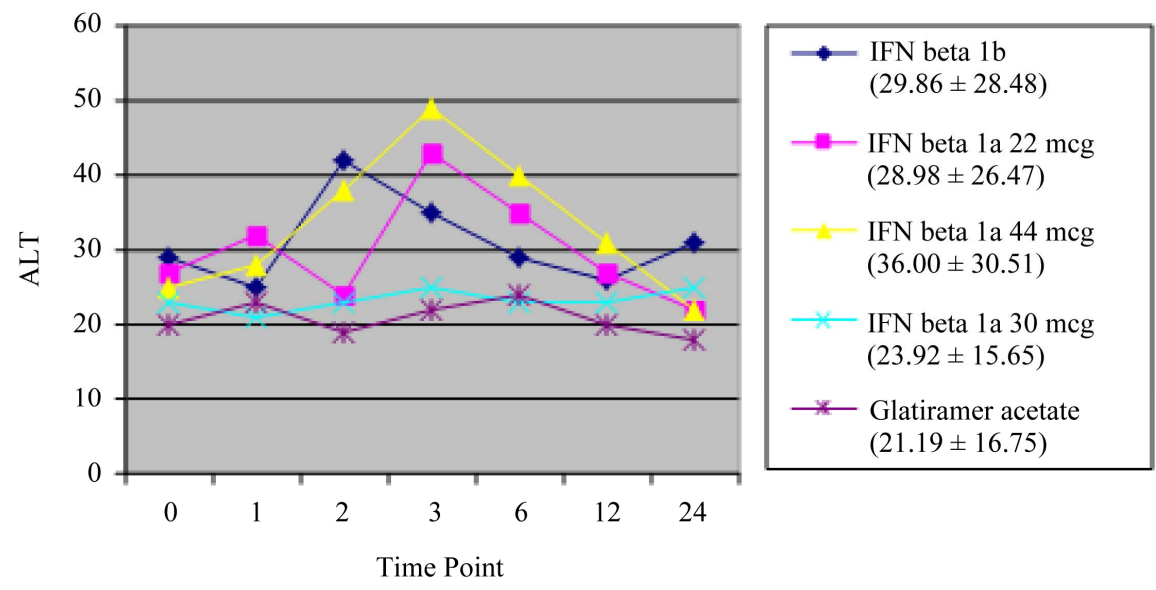

Time Point

Figure 4. ALT. Transient increase statistically significant for IFN beta $1 \mathrm{~b}(\mathrm{p}<0.001)$, IFN beta $1 \mathrm{a} 22 \mathrm{mcg}$ and $44 \mathrm{mcg}(\mathrm{p}<$ 0.01). Mean and SD of ALT values in brackets.

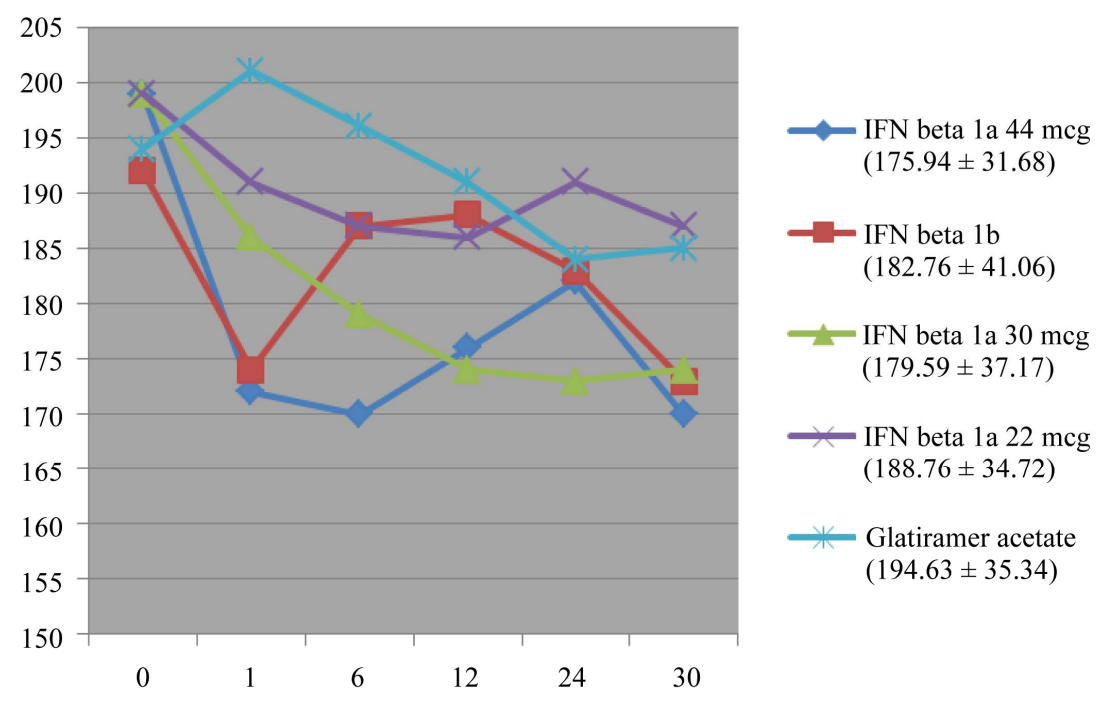

Figure 5. Total cholesterol level (mg/dl). Decrease of total cholesterol levels with a significant treatment effect for IFN beta $1 b$ $(\mathrm{p}<0.01)$, IFN beta 1 a $30 \mathrm{mcg}$ and IFN beta $1 \mathrm{a} 44 \mathrm{mcg}(\mathrm{p}<0.001)$, and a non significant trend with IFN beta $1 \mathrm{a} 22 \mathrm{mcg}$. 
Uric acid did not show significant changes during therapy.

Regarding peripheral blood counts, white blood cells (WBC) decreased significantly in treatment at month 3 $\left(6.04 \times 10^{3} / \mathrm{dl}, \mathrm{p}<0.001\right)$ and remained below baseline.

\section{Discussion}

The study of biochemical alterations in patients with MS has a historical value. It was one of the few tools to investigate this disease in last century, in the pre-MRI era, giving rise to pathogenetical hypothesis, such as the acid linolenic deprivation hypothesis [20]. More recently some have taken interest in blood chemistry analysis as diagnostic marker for MS, through specific measures of autoantibodies [21]. In the last years, blood analysis in MS natural history have been performed mainly to obtain more information relatively to the multifactorial pathogenesis of such a disease, looking for more effective therapies or combination therapies.

Lipids too have been largely investigated, and their study, also if not completely clarifying the primary or secondary role of their alteration in MS, has, anyhow, addressed the research towards statins mechanism of action in this disease. The use of such a class of drugs has been recognized effective as add-on therapy in patients not satisfactorily performing on IFNs alone $[22,23]$.

Our data confirm that in the course of MS there are some biochemical alterations, mainly due to the co-morbidity with other autoimmune disorders, but also to other mechanisms and in some cases even secondary to the disease itself. The secondary effects of the disease modifying treatments, analyzed in our cohort of patients, even if not clinically relevant were evident for all of the drugs, with some differences: we found a more pronounced alteration of liver enzymes and, with an inversion of tendency of the cholesterol levels, for the IFNs, with a dose dependent mechanism, and more evident effect on antithyroid antibodies production in the Glatiramer acetate group. This data is unexpected, since this drug is not related to autoantibodies production, but we cannot exclude that the production of anti-thyroid antibodies was more significant in this treatment group because of a bias in the treatment assignment: a history of previous autoimmune disorders or of a familiarity for these diseases might have led to the Glatiramer acetate therapy choice in some cases.

\section{Conclusions}

From the data of the literature we took the message that one of the most interesting metabolite altered in the course of MS is uric acid: its renown deficit in MS deserves further pathogenetic elucidation aimed at future therapeutic strategies. Regarding the aspects of disease modifying therapies adverse effects, mainly hepatic injury and thyroid function alterations, from the data retrieved from literature and our clinical experience, we might extrapolate some general observations:

1) Monitoring of blood chemistry parameters under immunomodulatory therapies for MS is warranted in the first year, in particular for liver enzymes, thyroid antibodies, blood cells.

2) Those subjects with abnormal baseline parameters require monitoring in the longer term, since blood analysis can be altered even at 24 months of therapy.

3) Therapies considered as "low dose" are not free from hepatotoxic risks.

4) Production of anti thyroid antibodies should be monitored also during treatment with Glatiramer acetate, given the high incidence of clinically significant values of auto antibodies up to two years of therapy in our population.

\section{REFERENCES}

[1] J. H. Noseworthy, "Progress in Determing the Causes and Treatment of Multiple Sclerosis," Nature, Vol. 399, 1999, pp. A40-A47.

[2] L. Steinman, "Multiple Sclerosis: A Coordinated Immunological Attack against Myelin in the Central Nervous System," Cell, Vol. 85, No. 3, 1996, pp. 229-302.

[3] M. Tiberio, D. T. Chard, G. R. Altmann, et al., "Metabolite Changes in Early Relapsing-Remitting Multiple Sclerosis. A Two Year Follow-Up Study," Journal of Neurology, Vol. 253, No. 2, 2006, pp. 224-230.

[4] C. Behl, "Oxidative Stress in Alzheimer's Disease: Implications for Prevention and Therapy," Sub-Cellular Biochemistry, Vol. 38, 2005, pp. 65-78.

doi:10.1007/0-387-23226-5 3

[5] Z. Gu, T. Nakamura, D. Yao, Z. Q. Shi, et al., "Nitrosative and Oxidative Stress Links Dysfunctional Ubiquitination to Parkinson's Disease," Cell Death and Differentiation, Vol. 12, No. 9, 2005, pp. 1202-1204. doi:10.1038/sj.cdd.4401705

[6] H. Tremlett and J. Oger, "Hepatic Injury, Liver Monitoring and the Beta-Interferons for Multiple Sclerosis," Journal of Neurology, Vol. 251, No. 11, 2004, pp. 1297 1303. doi:10.1007/s00415-004-0619-5

[7] L. Durelli, B. Ferrero, A. Oggero, et al., "Betaferon Safety Trial (BEST) Study Group. Liver and Thyroid Function and Autoimmunity during Interferon-Beta $1 \mathrm{~b}$ Treatment for MS," Neurology, Vol. 57, No. 8, 2001, pp. 13631370 .

[8] G. Niederwieser, W. Buchinger, R. M. Bonelli, et al., "Prevalence of Autoimmune Thyroiditis and Non-Immune Thyroid Disease in Multiple Sclerosis," Journal of Neurology, Vol. 250, No. 6, 2003, pp. 672-675. doi:10.1007/s00415-003-1053-9

[9] F. Giubilei, G. Antonini, S. Di Legge, et al., "Blood Cholesterol and MRI Activity in First Clinical Episode Sug- 
gestive of Multiple Sclerosis," Acta Neurologica Scandinavica, Vol. 106, No. 2, 2002, pp. 109-112. doi:10.1034/j.1600-0404.2002.01334.x

[10] S. Sotgiu, M. Pugliatti, A. Sanna, et al., "Serum Uric Acid and Multiple Sclerosis," Neurological Sciences, Vol. 23, No. 4, 2002, pp. 183-188. doi:10.1007/s100720200059

[11] G. Toncev, B. Milicic, S. Toncev, et al., "Serum Uric Acid Levels in Multiple Sclerosis Patients Correlate with Activity of Disease and Blood-Brain Barrier Dysfunction," European Journal of Neurology, Vol. 9, No. 3, 2002, pp. 221-226. doi:10.1046/j.1468-1331.2002.00384.x

[12] G. Toncev, B. Milicic, S. Toncev and G. Samardzic, "High-Dose Methylprednisolone Therapy in Multiple Sclerosis Increases Serum Uric Acid Levels," Clinical Chemistry and Laboratory Medicine, Vol. 40, No. 5, 2002, pp. 505-508.

[13] T. Vial and J. Descotes, "Clinical Toxicity of the Interferons," Drug Safety, Vol. 10, No. 2, 1994, pp. 115-150. doi:10.2165/00002018-199410020-00003

[14] F. Monzani, N. Caraccio, A. Casolaro, F. Lombardo, G. Moscato, L. Murri, E. Ferrannini and G. Meucci, "LongTerm Interferon Beta-1b Therapy for MS: Is Routine Thyroid Assessment Always Useful," Neurology, Vol. 55, No. 4, 2000, pp. 549-552.

[15] P. Rieckmann, P. O'Connor, G. S. Francis, G. Wetherill and E. Alteri, "Haematological Effects of InterferonBeta-1a (Rebif) Therapy in Multiple Sclerosis," Drug Safety, Vol. 27, No. 10, 2004, pp. 745-756. doi:10.2165/00002018-200427100-00005

[16] P. V. Byskosh and A. T. Reder, "Interferon Beta-1b Effects on Cytokine mRNA in Peripheral Mononuclear Cells in Multiple Sclerosis," Multiple Sclerosis, Vol. 1, No. 5, 1996, pp. 262-269.
[17] C. S. Constantinescu, P. Freitag and L. Kappos, "Increase in Serum Levels of Uric Acid, an Endogenous Antioxidant, under Treatment with Glatiramer Acetate for Multiple Sclerosis," Multiple Sclerosis, Vol. 6, No. 6, 2000, pp. 378-381.

[18] V. B. Morra, G. Coppola, G. Orefice, et al., "InterferonBeta Treatment Decreases Cholesterol Plasma Levels in Multiple Sclerosis Patients," Neurology, Vol. 62, No. 5, 2004, pp. 829-830.

[19] G. Coppola, R. Lanzillo, C. Florio, et al., "Long-Term Clinical Experience with Weekly Interferon Beta-1a in Relapsing Multiple Sclerosis," European Journal of Neurology, Vol. 13, No. 9, 2006, pp. 1014-1021. doi:10.1111/j.1468-1331.2006.01422.x

[20] R. W. Baker, H. Sanders, R. H. Thompson, et al., "Serum Cholesterol Linoleate Levels in Multiple Sclerosis," Journal of Neurology, Neurosurgery \& Psychiatry, Vol. 28, No. 3, 1965, pp. 212-217.

[21] F. Lolli, B. Mazzanti, M. Pazzagli, et al., "The Glycopeptide CSF114 (Glc) Detects Serum Antibodies in Multiple Sclerosis," Journal of Neuroimmunology, Vol. 167, No. 1-2, 2005, pp. 131-137. doi:10.1016/j.jneuroim.2005.05.016

[22] R. Lanzillo, G. Orefice, M. Quarantelli, et al., "Atorvastatin Combined to Interferon to Verify the Efficacy (ACTIVE) in Relapsing Remitting Active Multiple Sclerosis Patients: A Longitudinal Controlled Trial of Combination Therapy," Multiple Sclerosis, Vol. 16, No. 4, 2010, pp. 450-454. doi:10.1177/1352458509358909

[23] M. Togha, S. A. Karvigh, M. Nabavi, et al., "Simvastatin Treatment in Patients with Relapsing-Remitting Multiple Sclerosis Receiving Interferon Beta 1a: A Double-Blind Randomized Controlled Trial," Multiple Sclerosis, Vol. 16, No. 7, 2010, pp. 848-854. doi: $10.1177 / 1352458510369147$ 